\title{
Image splicing detection method based on particle swarm optimization (PSO) algorithm
}

\author{
Gan Ling ${ }^{1}$, Liu Xiao, a and Zou Kuanzhong ${ }^{1}$ \\ ${ }^{1}$ College of Computer Science and technology, Chongqing University of Posts and \\ telecommunications, Chongqing 400065, China \\ aliuxiao_2015y@163.com
}

Keywords: splicing detection, support vector machine (SVM), particle swarm optimization (PSO)

\begin{abstract}
Splicing is copy-move operation on different image, and it is one of the most commonly used operations of image tampering. Aiming at the poor performance on detection, a kind of image splicing detection method based on particle swarm algorithm is proposed. First, we extracted two different image features: Markov and gray level co-occurrence matrix (GLCM) in the image database, which are used to verify the effectiveness of the particle swarm algorithm on different features. Then, we normalized the extracted image features to reduce the impact of identification results which comes from the singular value. Finally, the optimal SVM classification model was obtained by using the particle swarm optimization algorithm. Experiment on the two different features of the image database of Columbia University shows that the image splicing detection method based on particle swarm can improve the detection rate on different features, and achieve the best recognition rate of 93.20\% on Markov features.
\end{abstract}

\section{Introduction}

The user-friendly and powerful image processing software has appeared to beautify people's life due to the development of digital technology. However, the malicious tampering with image has caused many negative effects to the society in these days. Image matching is one of the most common ways of image tampering and it has become a research hotpot.

Many research results about the image splicing have been achieved by the researches all around the world. Shi [1] for the first time to apply Markov features in the field of digital image splicing detection, combined with torque characteristic features, through the RBF kernel function of SVM classification, the experimental use of Columbia University stitching image database [2], the best recognition rate is $91.87 \%$. He [3] used multistep Markov features in DCT and DWT domain applications, through the dimensionality of the SVM parameters optimization, achieve detection rate to $93.55 \%$. In addition, He [4] using the approximate length of run features, such as on the gray image database have achieved $80.58 \%$ detection rate, this method reduces the computational complexity. Saba [5] using the gray level run length matrix features, and on the CASIA database has a detection rate of $80.71 \%$. WANG WEI [6] proposed a color image splicing detection method based on the colorimetric analysis, using the gray level co-occurrence matrix image for image segmentation, edge detection experiments using stitching image color images at Columbia University library, which has achieved the best recognition rate is $90.5 \%$ on $\mathrm{Cr}$ chromaticity. Besides, some other method based on human visual system, multi-level classification model [7] and the method based on edge fuzzy metric [8] have been reported.

In this paper, a kind of image splicing detection method based on particle swarm optimization (PSO) algorithm has been proposed. Numerical simulation is performed to validate the performance of the proposed technology. The method using particle swarm optimization(PSO) algorithm in detecting part, established optimization support vector machine (SVM) classification model, so as to improve the accuracy of splicing detection. 
The rest of the paper is organized in the following sequence. In Section 2 and 3, the proposed technology is introduced in detail. In Section 4, numerical simulation results are made and given to demonstrate the validity of the method. Concluding remarks are summarized in the final section.

\section{The splicing detection algorithm}

Figure 1 is a digital image splicing detection basic flow chart of the algorithm. The first step is to existing image library is divided into two parts: training image library classification model (training) and test image library (effect) test algorithm, and then extract the image feature; The last image splicing detection.

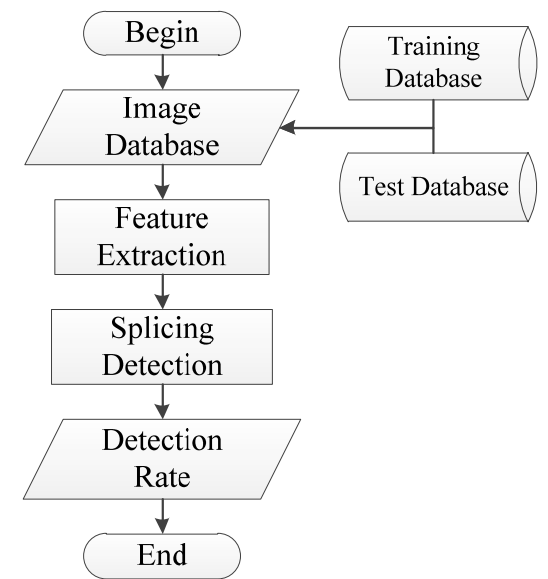

Fig. 1. The flowchart of splicing detection algorithm

Digital image splicing detection algorithm process step is crucial in image feature extraction, image features, the existing Markov features and features of GLCM is two kinds of features of the application scope is wide.

Markov features. Markov features reflect the state transition probability of image information, this information can reflect the change of the correlation between image pixels, and image splicing can cause the change. The flowchart of extracting the features of Markov process is shown in figure 2.

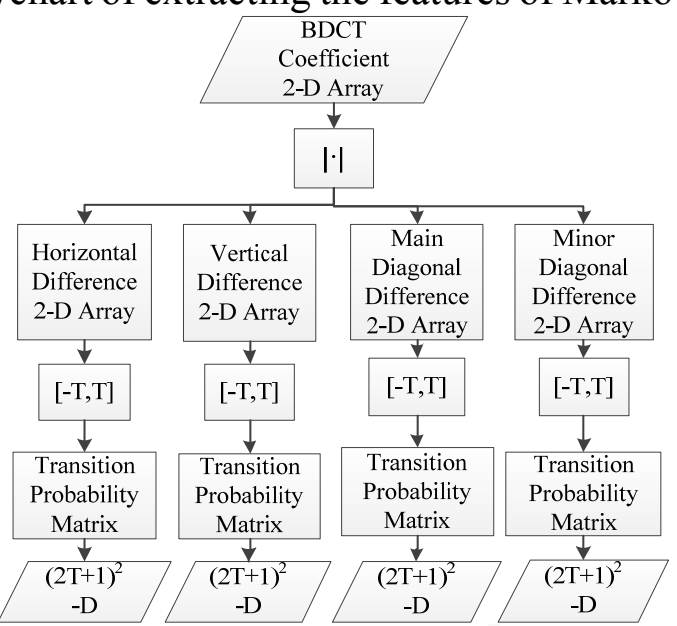

Fig. 2. The flowchart of To extracting features of Markov

The first step for block BDCT coefficient matrix of the image, the BDCT coefficient matrix after the integer of the horizontal and vertical difference of two directions, horizontal difference matrix and vertical difference matrix, the calculation formula as shown in Eq.1 and Eq.2.

$$
\begin{aligned}
& F_{h}(\mu, v)=F(\mu, v)-F(\mu+1, v) \\
& F_{v}(\mu, v)=F(\mu, v)-F(\mu, v+1)
\end{aligned}
$$

Last two direction of horizontal and vertical difference matrix calculation of the corresponding state transition probability matrix of the horizontal and vertical direction, and matrix deformation of 
the row vector as the Markov features of the image. The calculation formulas are shown as Eq. 3 and Eq.4.

$$
\begin{aligned}
& p\left\{F_{h}(\mu+1, v)=n \mid F_{h}(\mu, v)=m\right\}=\frac{\sum_{v=0}^{S_{v}-2} \sum_{\mu=0}^{S_{\mu}-2} \delta\left(F_{h}(\mu, v)=m, F_{h}(\mu+1, v)=n\right)}{\sum_{v=0}^{S_{v}-2} \sum_{\mu=0}^{S_{\mu}-2} \delta\left(F_{h}(\mu, v)=m\right)} \\
& p\left\{F_{v}(\mu, v+1)=n \mid F_{v}(\mu, v)=m\right\}=\frac{\sum_{v=0}^{S_{v}-2} \sum_{\mu=0}^{S_{\mu}-2} \delta\left(F_{v}(\mu, v)=m, F_{v}(\mu, v+1)=n\right)}{\sum_{v=0}^{S_{v}-2} \sum_{\mu=0}^{S_{\mu}-2} \delta\left(F_{v}(\mu, v)=m\right)}
\end{aligned}
$$

Image stitching operation will also change the image texture information, therefore GLCM feature [5] can be used as the basis of splicing detection.

GLCM features. GLCM is gray level co-occurrence matrix. It is image grey statistics, can reflect the direction of image gray level and change of information. GLCM feature is extracted based on gray level co-occurrence matrix of ASM (second moment), COR (autocorrelation), CON (contrast) and ENT (entropy), four unrelated information. The calculation formulas are shown as Eq.5 to Eq.8.

$$
\begin{aligned}
& A S M=\sum_{i=1}^{k} \sum_{j=1}^{k}(G(i, j))^{2} \\
& C O R=\sum_{i=1}^{k} \sum_{j=1}^{k} \frac{(i j) G(i, j)-u_{i} u_{j}}{s_{i} S_{j}} \\
& C O N=\sum_{n=0}^{k-1} n^{2}\left\{\sum_{|i-j|=n} G(i, j)\right\} \\
& \text { ENT }=-\sum_{i=1}^{k} \sum_{j=1}^{k} G(i, j) \log G(i, j)
\end{aligned}
$$

Finally the GLCM feature is composed of more than four unrelated texture information characteristic vector and the features are as follows

$$
G L C M=\{A S M, C O R, C O N, E N T\}
$$

Support vector machine (SVM). Digital image splicing detection is a binary classification problems, the two classes are real images and spliced images. Each image as a sample, it is the first eigenvector of the image, for the image tag (for real images, for stitching images). $g(x)=w^{T} x+w_{0}$ is assuming the classification hyper-plane, modeling formula is as follows follow

$$
\min S\left(w, w_{0}, \xi\right)=\frac{1}{2}\|w\|^{2}+C \sum_{i=1}^{N} \xi_{i}
$$

and the constraint is follow

$$
\left\{\begin{array}{l}
y_{i}\left(w^{T} x_{i}+w_{0}\right) \geq 1-\xi_{i} \\
\xi_{i} \geq 0
\end{array}\right.
$$

where $C \geq 0,0<\xi_{i} \leq 1$. And we use the classifier is open source software package LIBSVM SVM [11].

\section{The particle swarm algorithm}

In this paper, for the first time the particle swarm algorithm (PSO) is applied to the field of digital image splicing detection. Particle swarm algorithm can fast convergence, and its global optimization 
ability is stronger, simpler implementation. Relation to other optimization algorithms, PSO algorithm is more suitable for image splicing detection. Its computation complexity is relatively low.

Unlike other optimization algorithms, PSO algorithm is first random initial search point, and the optimal value through the update mechanism to update the individual and the global optimal value. PSO algorithm flow chart is shown in figure 3.

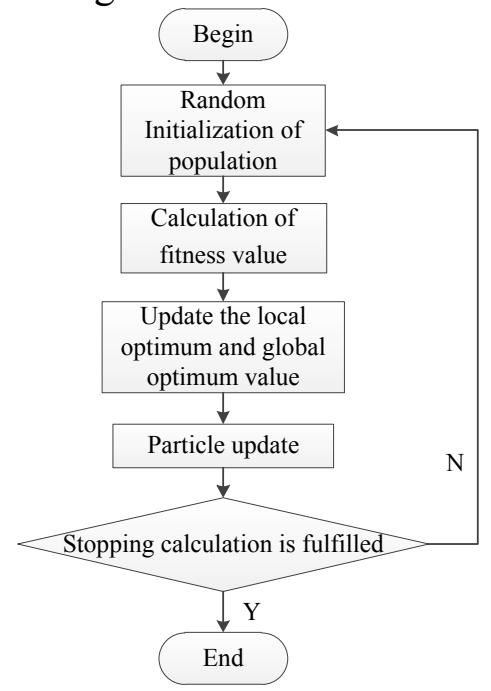

Fig. 3. The flowchart of PSO algorithm

Particles update based on the formula as Eq.12 and Eq.13.

$$
\begin{gathered}
v_{i j}^{t+1}=\omega \times v_{i j}^{t}+c_{1} \times \operatorname{rand}() \times\left(p_{i j}-x_{i j}^{t}\right) \\
+c_{2} \times \operatorname{rand}() \times\left(p_{g j}-x_{i j}^{t}\right) \\
x_{i j}^{t+1}=x_{i j}^{t}+v_{i j}^{t+1}
\end{gathered}
$$

Where $\omega$ is inertia weight, rand () is a randomly generated $(0,1)$ within the scope of the function is real number and $c_{1} 、 c_{2}$ learning factor.

Finally, the optimized by PSO algorithm can find the most suitable for this paper, the SVM classifier radius of penalty factor $\mathrm{C}$ and kernel function $\mathrm{g}$.

\section{The simulation results and analysis}

In calculation, we use a computer with Core i5-3470 CPU 3.2 GHz and 4096 Mbytes memory under Windows 7 system. Experimental image library USES the Columbia image splicing database [2].

Columbia image splicing database [2] is currently the most widely used image splicing detection field of an open source database. In this paper, the experiment using the gray scale image database. The database has a piece of real images of 933 and 912, the image size is $128 * 128$, the format is BMP. All have no image smoothing, edge blur and subsequent image processing operations.

To meet the demand of experiment, this article will gray image images library database is divided into training and test image library. Training by $5 / 6$ of the real image library and $5 / 6$ of the Mosaic image of test image library by a real images of the rest of the 1/6 and 1/6 of the Mosaic image.

In this paper, we do experiments in Markov transition probability (Markov) [1] and features of gray level co-occurrence matrix (GLCM) [6] on the basis of comparison respectively, in order to verify the validity of the algorithm. Contrast experiment using three evaluation indexes: correct recognition Accuracy of the real image TP (true positive), correct recognition image mosaics of TN Accuracy (true negative) and the correct recognition Accuracy of two types of image Accuracy. 
Markov features based on the contrast experiment results. Figure 4 shows the Markov characteristic curve of the fitness of this method. The number of iterations is 100 , the populations is 20 .

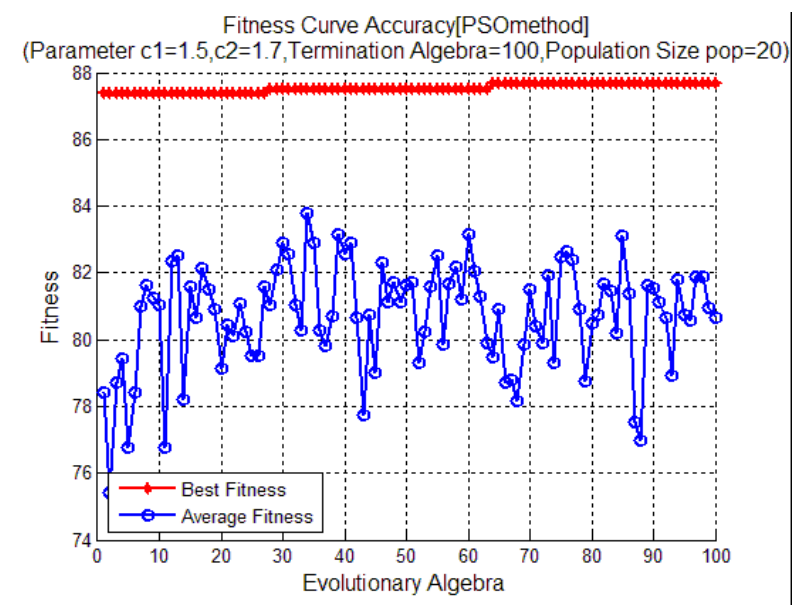

Fig. 4. The curve of Markov characteristic fitness

Table 1 illustrates of contrast experiment results based on the Markov features.

Table.1.The contrast experiment results based on the Markov features

\begin{tabular}{cccc}
\hline & $\mathrm{TP} / \%$ & $\mathrm{TN} / \%$ & Accuracy/\% \\
\hline The orignial algorithm $^{[1]}$ & 86.61 & 90.03 & 88.31 \\
\hline The proposed algorithm & 94.87 & 91.50 & 93.20 \\
\hline Variation & +8.26 & +1.47 & +4.89 \\
\hline
\end{tabular}

It can be seen from the comparison in table 2, the method in real image Accuracy than the original algorithm (TP), splicing image Accuracy (TN) Accuracy were increased and the Accuracy improved by $4.89 \%$.

GLCM features based on the contrast experiment results. Figure 5 shows the GLCM characteristic curve of the fitness of this method. The number of iterations is 100, the populations is 20 .

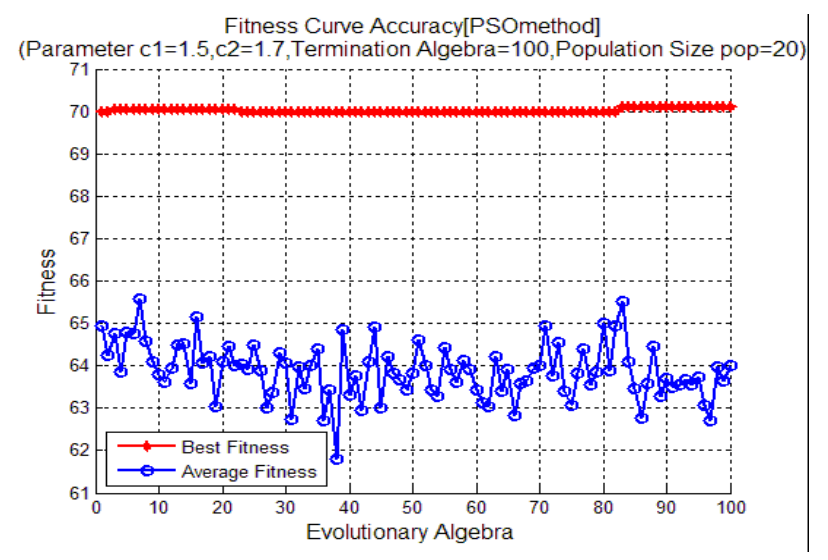

Fig. 5. The curve of GLCM characteristic fitness

Table 2 illustrates of contrast experiment results based on the GLCM features.

Table.2.The contrast experiment results based on the GLCM features

\begin{tabular}{cccc}
\hline & $\mathrm{TP} / \%$ & $\mathrm{TN} / \%$ & Accuracy $/ \%$ \\
\hline The orignial algorithm $^{[1]}$ & 53.21 & 65.35 & 59.22 \\
\hline The proposed algorithm & 60.26 & 75.81 & 67.96 \\
\hline Variation & +7.05 & +10.46 & +8.74 \\
\hline
\end{tabular}

It can be seen from the comparison table 3, the method than the original algorithm in $\mathrm{TP}, \mathrm{TN}$ and the effect on the Accuracy were improved obviously, especially TN increased by $10.46 \%$. 


\section{Summary}

In this paper, a splicing detection method based on particle swarm optimization (PSO) algorithm has been proposed to overcome the shortcomings of the existing image splicing detection methods. On the different features of algorithm in this paper has carried on the experiment, the experimental results show that this method can effectively improve the recognition rate of the image splicing detection, and has universal applicability in different features. Some numerical experiments are made to test the validity and capability of the proposed technology. Furthermore, the proposed algorithm is also relatively increase the complexity of the algorithm, therefore, reduce the complexity of the algorithm is the next job.

\section{References}

[1] SHI YUNQING, CHEN CHUNHUA, CHEN WEN. A natural image model approach to splicing detection [C]. MM\&Sec: Proceeding of workshop on Multimedia and security. New York: ACM. 2007: 51-62.

[2] Columbia DVMM Research Lab. (2004) Columbia Image Splicing Detection Evaluation Dataset. $\quad$ [DB/OL].

[DB/OL]. [2015-09-13]. http://www.ee.columbia.edu/ln/dvmm/downloads/AuthSplicedDataSet/AuthSplicedDataSet.ht m.

[3] HE ZHONGWEI, LU WEI, SUN WEI/et al. Digital image splicing detection based on Markov features in DCT and DWT domain [J]. Pattern Recognition, 2012, 45(12): 4292-4299.

[4] HE ZHONGWEI, LU WEI, SUN WEI/et al. Digital image splicing detection based on approximate run length [J]. Arm Rognon Lr, 2011(12):1591-1597.

[5] SABA MUSHTAQ, AJAZ HUSSAIN MIR. Novel method for image splicing detection [C]. ICACCI: Advances in computing, communications and informatics international conference. New Delhi: IEEE. 2014:2398-2403.

[6] WAGN WEI, DONG JING, TAN TIENIU.Effective image splicing detection based on image chroma [C].ICIP:International conference on image processing.Cairo: IEEE. 2009:1257-1260.

[7] QU ZHENHUA, QIU GUOPING, HUANG JIWU. Detect Digital Image Splicing with Visual Cues [M]. Springer Berlin Heidelberg. 2009. 5806:247-261.

[8] ZHENG QIANRU, SUN WEI, LU WEI. Digital spliced image forensics based on edge blur measurement [C]. ICITIS:IEEE International Conference on Information Theory and Information Security. Beijing: IEEE. 2010:399-402.

[9] KENNEDY J, EBERHART R. Particle swarm optimization[C].IEEE International Conference on Neural Networks.Perth,Australia:IEEE. 1995:1942-1948.

[10] GALLOWAY MARY M. Texture analysis using gray level run lengths [J].Computer Graphics \& Image Processing, 1975, 4(2): 172-179.

[11] CHANG CHIH-CHUNG,LIN CHIH-JEN.LIBSVM: A library for support vector machines[J]. ACM Transactions on Intelligent Systems and Technology.2006,2(3):389-396. 